\title{
Special issue of ADAM devoted to the International Workshop on Symmetries of Graph and Networks 2018
}

We are delighted to present this special issue of the Art of Discrete and Applied Mathematics (ADAM), on topics presented or related to topics covered at the TSIMF workshop on 'Symmetries of Graphs and Networks', held at Sanya, on the beautiful semi-tropical island province of Hainan (China), in January 2018.

This workshop added to the series of conferences and workshops on symmetries of graphs and networks initiated at BIRS (Canada) in 2008 and progressed in Slovenia every two years from 2010 to 2016.

It was attended by 50 mathematicians from China and other parts of the world (including Australia, Canada, New Zealand, Slovakia, Slovenia, South Korea and the USA), many of whom gave lectures on a range of topics involving the symmetries of graphs and maps, including Cayley graphs, arc-transitive graphs and digraphs, covering graphs, regular maps on surfaces, and regular Cayley maps, plus related topics such as graph embeddings and skew morphisms of groups.

Participants very much enjoyed the venue, which is similar in style to the BIRS facilities in Banff and Oaxaca and the institute at Oberwolfach, giving plenty of opportunity for interactions between participants, and stimulating further research on the topics covered.

This issue contains a number of interesting papers resulting from or associated with the workshop. We would like to thank the authors for their valuable contributions. Also we are very grateful to the TSIMF for its generous financial support and administrative assistance for the workshop, and to the chief editors of ADAM for making this special issue possible.

Marston Conder (University of Auckland, New Zealand) and Yan-Quan Feng (Beijing Jiaotong University, China)

Principal organisers of the workshop and Editors of this issue 